\title{
Factors and Economic Outcomes Associated with Documented Difficult Intubation in the United States
}

This article was published in the following Dove Press journal:

ClinicoEconomics and Outcomes Research

\author{
Marilyn A Moucharite (D) \\ Jianying Zhang (D) \\ Robert Giffin
}

Healthcare Economics Outcomes Research, Medtronic, Mansfield, MA, USA
Correspondence: Marilyn A Moucharite Medtronic, 15 Hampshire Street, Mansfield, MA, 02048, USA

Tel + I 774-284-5248 Email Marilyn.A.Moucharite@medtronic. com
Purpose: Establishing good mechanical ventilation is a critical component and prerequisite to a wide range of surgical and medical interventions. Yet difficulties in intubating patients, and a variety of associated complications, are well documented. The economic burden resulting from difficult intubation (DI), however, is not well understood. The current study examines the economic burden of documented DI during inpatient surgical admissions and explores factors that are associated with DI.

Patients and Methods: Using data from the Premier Healthcare Database, adult patients with inpatient surgical admissions between January 1, 2016 and December 31, 2018 were selected. International Classification of Diseases, Tenth Edition, Clinical Modification (ICD-10-CM) diagnosis codes were used to classify the patients into matched cohorts of DI and non-DI patients. Results: Patients in the DI group have mean inpatient costs and intensive care unit (ICU) costs that are substantially higher than patients without difficult intubations $(\$ 14,468$ and $\$ 4,029$ higher, respectively). Mean hospital length of stay and ICU length of stay were 3.8 days and 2.0 days longer, respectively (all $\mathrm{p}<0.0001$, except ICU cost $\mathrm{p}=0.0001$ ) in the DI group. Obesity, other chronic conditions, and larger hospital size were significantly associated with DI.

Conclusion: DI is associated with higher average cost and longer average length of stay. Keywords: cost burden, difficult intubation, airway management, health economics

\section{Plain Language Summary}

In this study, we compared the difference in hospital cost and length of hospital stay between two groups of patients. Both groups of patients were admitted to the hospital because of the need for a surgical procedure. When an anesthesiologist inserts a tube into the airway to ventilate a patient before administering anesthesia, the anesthesiologist might encounter some patients for whom the tube is difficult to insert. Patients with this characteristic were designated to the "difficult intubation" (DI) group. Patients without this characteristic were designated to the "non-difficult intubation" (non-DI) group. We made sure these two groups of patients were comparable in terms of similar age, gender, and disease conditions, and stayed in similar types of hospitals and had similar procedures. We found that when we compared the DI group to the non-DI group, on average, the hospital cost was $\$ 14,468$ more and length of hospital stay was 3.8 days longer for the DI group. This study highlights the significant cost burden associated with difficult intubation and suggests that further research to enhance clinicians' ability to predict DI would be beneficial.

\section{Introduction}

During tracheal intubation, an anesthetist inserts a tube through the mouth or nose and down the trachea into the lungs. This is a common procedure for patients who 
undergo surgical procedures. Difficult intubation (DI), which increases the number of laryngoscopic attempts during tracheal intubation, has been associated with a variety of complications including oxygen desaturation, hypertension, dental damage, admission to the intensive care unit (ICU), and complications at extubation, as well as arrhythmias, bronchospasm, airway trauma, CICV ("can't intubate, can't ventilate"), and sequela of hypoxia (cardiac arrest, brain damage, and death). ${ }^{1}$

Many factors influence the success rate of intubation. Studies have shown that the first-pass success rate of intubation in the operating room ranges from only $63 \%$ to $85 \%{ }^{2}$ Up to $93 \%$ of difficult intubations are unanticipated. ${ }^{3}$ Patient risk factors, such as restrictions on neck movement, mouth opening, mallampati class ${ }^{4}$ and thyromental distance and neck extension are common factors. ${ }^{5}$ Many studies have tried to establish the best and most feasible method to predict difficult intubation, including Cormack-Lehane classification system $^{6}$ and the Intubation Difficulty Scale (IDS). ${ }^{7}$ One study of experienced anesthesiologists, utilizing the widely used Cormack-Lehane classification system, found they did not score the same patients consistently over time, and that only $25 \%$ could correctly define all four grades of the CormackLehane classification system. ${ }^{8}$ Under certain emergency circumstances (e.g., severe head trauma or suspected cervical spine injury), it may be impossible to fully utilize these various classification systems during physical examination to predict the difficulty of tracheal intubation. ${ }^{9}$

Before the advent of the ICD-10-CM coding system (International Classification of Diseases, Tenth Revision, Clinical Modification), there was no mechanism to quantify the cost burden of failed or difficult intubation from medical claims data. The implementation of ICD-10-CM introduced codes specific to DI, which enables researchers to identify cases with documented DI during surgical procedures. To our knowledge, there is no study currently available that examines the cost and healthcare utilization outcomes associated with difficult intubation in the hospital operating room setting. We utilized the ICD-10-CM coding system to classify patients into two groups, those with and without DI, to conduct a retrospective matched cohort study to fill this knowledge gap. This study will shed light from a hospital provider economic perspective on the consequence of DI in the inpatient hospital setting. The objective of this study was two-fold: First, to examine the healthcare utilization and cost associated with DI during an inpatient surgical procedure; and second, to explore the major patient characteristics and hospital characteristics of cases that were reported as DI.

\section{Patients and Methods \\ Construction and Content}

A retrospective observational cohort study was conducted using three years of data (2016-2018) from the Premier Healthcare Database ${ }^{\circledR}$ (Premier, Inc., Washington, D.C.).

\section{Data Source}

The Premier ${ }^{\circledR}$ Database contains data from more than 600 United States (US) hospitals and includes de-identified Universal Billing (UB-04) data for approximately 1 out of every 5 discharges in the U.S. ${ }^{10}$ It also includes information for each discharge describing patient demographics, hospital characteristics, payer information, Medicare Severity-Diagnosis Related Group (MS-DRG), ICD-10-CM primary and secondary diagnosis and procedure codes, admission and discharge calendar quarter and year, and detailed hospital department-level billing and cost data for each discharge. As an administrative database containing de-identified data, institutional review board or ethics committee approval was not required for this study. Cost data represent the actual total costs to treat the patient and include both fixed and variable costs. ${ }^{10}$ Approximately $60 \%$ of hospitals in the Premier database report costs. For the remaining hospitals, costs are calculated by Premier using charges reported to Premier and department-specific cost-to-charge ratios reported by hospitals to the Centers for Medicare \& Medicaid Services.

\section{Cohort Selection}

All adult patients who underwent inpatient surgical procedures between January 1, 2016 and December 31, 2018 were included in the initial cohort selection. Patients with difficult intubation (DI group) were identified using three ICD-10 codes on the discharge record, which correspond to "failed or difficult intubation" on an initial encounter (T88.4XXA), subsequent encounter (T88.4XXD), or sequela (T88.4XXS). The following patients were excluded from the study: 1) Pregnant women; 2) Cases where general anesthesia was not indicated in the master charge file; 3) Patients with a primary procedure that indicates the Major Diagnostic Category (MDC) was a) not documented, b) "Unrelated Operating Room Procedures" or c) "Invalid or Ungroupable MS-DRG"; and 4) "Pre-MDC" which included all organ transplants 
or tracheotomies. These exclusion criteria applied to both the DI group and the non-DI group. In addition, the DI group excluded patients with diagnosis code indicating failed or difficult intubation (ICD-10 codes: T88.4XXA, T88.4XXD, T88.4XXS) was present on admission and the non-DI group excluded patients with primary procedures that were not present in the DI group.

Pregnant women were excluded from the study because the level of difficulty of intubation and procedure type likely differ for pregnant women compared to nonpregnant adults. Admissions with primary procedures not in the DI group were excluded because the risk adjustment process includes an exact match on primary procedure as well as a match on propensity score between the DI and non-DI groups to ensure that cost difference is based on comparison of cases with the same primary procedure. The reasons for implementing the remaining exclusion criteria were to make the cohort groups comparable by ensuring all patients in the study underwent general anesthesia during a surgical procedure, all cases were inpatient admissions, and all patients in the DI group experienced difficult intubation during rather than prior to the admission.

Multiple steps were applied to identify the comparison group, non-DI, and implement risk adjustment to confirm comparison of demographically and clinically similar patients. The risk adjustment process involved two steps:

1. Exact match between DI and non-DI patients on primary surgical procedures: to qualify for the nonDI group, patients must have the same primary procedure, identified using the ICD-10-PCS (International Classification of Diseases, Tenth Revision, Procedure Coding System) code, as in the DI group (Supplementary Table 1).

2. One to one DI vs non-DI propensity score matching within each primary procedure. The matching process between the DI and non-DI groups matched cases within each primary procedure based on propensity scores using a nearest neighbor matching method. The propensity score was calculated using a multivariable logistic regression model which included as predictor variables both patient characteristics (age group, gender, race, admission type (Urgent/Emergent or Elective), insurance type, comorbid conditions), and hospital characteristics (region of the country, teaching status, number of beds, urban/rural designation). The 12 comorbid conditions included in the propensity score model were obesity, cancer, congestive heart failure (CHF), chronic obstructive pulmonary disease (COPD), cardiovascular disease, diabetes, diabetes with sequela, hypertension, peripheral vascular disease, mild liver disease, moderate/ severe liver disease, and paralysis. These 12 comorbid conditions were selected from the 17 comorbid conditions that make up the Charlson Comorbidity Index (CCI) using a "purposeful selection" process.

The "purposeful selection" process of determining which predictor variables to include in a logistic regression model was developed by researchers at the University of Arkansas for Medical Sciences the and University of Massachusetts, Amherst ${ }^{11}$ and implemented for this research using an automated SAS macro developed by Bursac et al. ${ }^{12}$ The "purposeful selection" process begins with a univariate analysis of the candidate predictive variables, in this case the 17 comorbid conditions. The initial model includes variables with p-values that fall below the pre-defined threshold for statistical significance $(p<0.25)$. An iterative process adds the variables that were not initially found to be statistically significant into the model one at a time to evaluate each variable for statistical significance in the new model, using a p-value threshold of $\mathrm{p}<0.1$, and confounding, which is defined as a $15 \%$ change in a parameter estimate in the new model compared to the original model. The variables that are statistically significant or determined to be confounders are retained in the new model. This iterative process results in a final variable selection that includes significant variables and important confounding variables. ${ }^{11}$ The threshold values recommended by the authors of the "purposeful selection" process ${ }^{11}$ were used to determine which variables to include $-\mathrm{p}<0.25$ for inclusion in the initial model, $\mathrm{p}<0.1$ to remain in the model after being added back in, and $15 \%$ change in a predictor variable.

\section{Outcome Measures}

Primary outcome measures were average cost of admission, hospital length of stay (LOS), post-operative Intensive Care Unit (ICU) admission rate, cost of ICU stay, and length of ICU stay. Post-operative ICU was defined as ICU admissions that occurred on the same day of surgery or within two days post-surgery. Costs for admissions in 2016 and 2017 were adjusted to 2018 
dollars using the US Bureau of Labor Statistics Consumer Price Index for Medical Care.

\section{Statistical Analysis}

Descriptive univariate analysis was used to compare baseline patient demographics, clinical characteristics, and hospital characteristics between the DI and non-DI groups. Chi-squared test was used for categorical variables and the two sample $t$-test was used for continuous variables such as LOS or cost. A multivariable logistic regression model was used to calculate adjusted odds ratios (OR) to examine patient and hospital characteristics that are associated with documented DI. Variables modeled in the logistic regression included (1) patient characteristics: age group, gender, race, admission type (Urgent/Emergent or Elective), insurance type, comorbid conditions and (2) hospital characteristics: region, teaching status, number of beds, urban/rural designation. Statistical significance was defined as p-value $<0.05$. All statistical analyses were conducted using $\mathrm{SAS}^{\circledR}$ 9.4 for UNIX (SAS Institute Inc Cary, NC, USA).

This study did not directly involve human subjects. All patient data were de-identified and were compliant with the Health Insurance Portability and Accountability Act (HIPAA). Therefore, this study was exempt from Institutional Review Board (IRB) approval (45 CFR $\S$ 46.0001(b) (4)).

\section{Results}

\section{Characteristics of the DI and Non-DI Groups Before Propensity Score Matching}

A total of 2,233,751 cases met the eligibility criteria for the analysis. Of these, 609 cases were in the DI group and $2,233,142$ cases were in the non-DI group. Patient and provider characteristics, and economic outcomes associated with each cohort, are described in Figure 1 and Table 1.

Prior to matching, patients in the DI group were more likely be male ( $59 \%$ vs. $45 \%, \mathrm{p}<0.0001)$, black $(21 \%$ vs. $10 \%, \mathrm{p}<0.0001)$, less than 65 years old $(57 \%$ vs. $52 \%$, $\mathrm{p}=0.019)$ and have urgent or emergent admissions (54\% vs. $39 \% \mathrm{p}=<.0001$ ) (Table 1). Notably, patients in the DI group were more likely to have comorbidities such as obesity (44\% vs. $26 \%$ ), cancer (14\% vs. $9 \%$ ), CHF (22\% vs.12\%), COPD (29\% vs. $18 \%$ ) and renal disease (20\% vs.11\%), compared to patients in the non-DI group. All p-values for these comorbidities were $<0.0001$ (Table 2). Compared to patients in the non-DI group, patients who were treated in a teaching hospital or a large hospital (500 beds or more) were more likely to

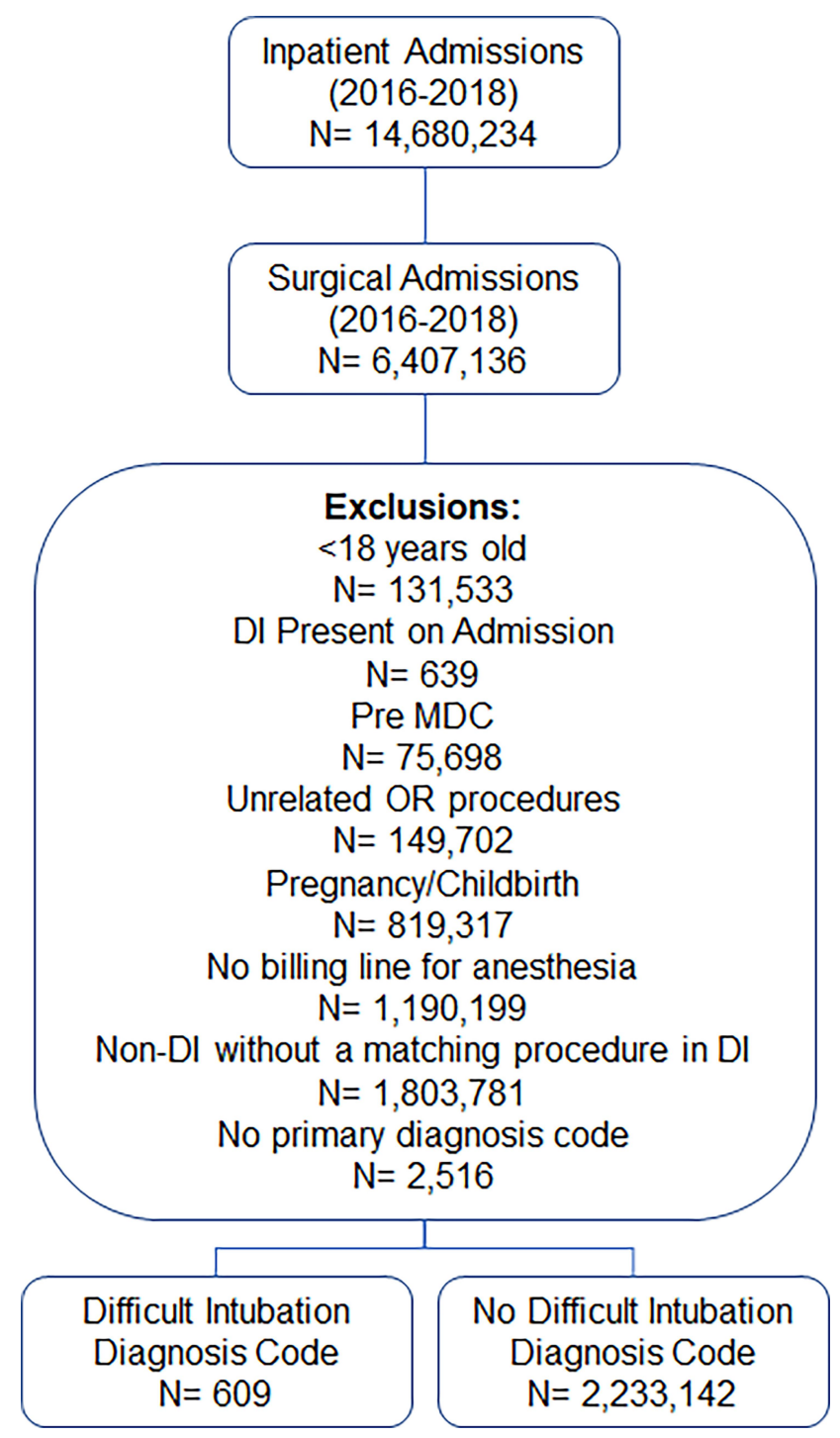

Figure I Patient selection criteria for inclusion in the study.

Abbreviations: DI, difficult intubation; MDC, major diagnostic category; OR, operating room.

be documented as DI patients, $54 \%$ vs. $47 \%(\mathrm{p}=0.0001)$ and $50 \%$ vs. $35 \%(\mathrm{p}<0.0001)$ respectively (Table 1$)$.

Patients in the DI group had significantly higher mean cost of hospital inpatient stay $(\$ 45,251 \pm \$ 43,613$ vs. $\$ 22,056 \pm \$ 27,008)$; longer $\operatorname{LOS}(10.7 \pm 12.1$ days vs. 4.5 \pm 5.8 days); higher post-operative ICU admission rate $(60 \%$ vs. $16 \%)$; higher ICU costs $(\$ 11,657 \pm \$ 15,653$ vs. $\$ 7,049 \pm$ $\$ 9,386)$ and longer length of stay in the ICU $(5.4 \pm 5.8$ days vs. $3.4 \pm 3.9$ days). All p-values were $<0.0001$ (Table 3 ).

\section{Results Based on Matched Cohorts of Patients}

Propensity score matching reduced the number of patients in each cohort to 606 (Table 3). The matching process 
Table I Patient and Hospital Demographic Characteristics and Logistic Regression Outputs Prior to Risk Adjustment Matching

\begin{tabular}{|c|c|c|c|c|c|c|c|c|c|}
\hline \multirow[t]{3}{*}{$\begin{array}{l}\text { Patient } \\
\text { Characteristics }\end{array}$} & \multirow{2}{*}{\multicolumn{2}{|c|}{$\begin{array}{c}\begin{array}{c}\text { Without Difficult } \\
\text { Intubation }\end{array} \\
2,233,142\end{array}$}} & \multirow{2}{*}{\multicolumn{2}{|c|}{$\begin{array}{c}\begin{array}{c}\text { With Difficult } \\
\text { Intubation }\end{array} \\
609\end{array}$}} & \multirow[t]{3}{*}{ p-value ${ }^{a}$} & \multirow{2}{*}{\multicolumn{4}{|c|}{$\begin{array}{c}\text { Comparing Difficult Intubation to No } \\
\text { Difficult Intubation }\end{array}$}} \\
\hline & & & & & & & & & \\
\hline & \multirow[t]{2}{*}{$\begin{array}{l}\text { Number of } \\
\text { Patients }\end{array}$} & $\begin{array}{l}\text { Percent of } \\
\text { Patients }\end{array}$ & $\begin{array}{l}\text { Number of } \\
\text { Patients }\end{array}$ & $\begin{array}{l}\text { Percent of } \\
\text { Patients }\end{array}$ & & $\begin{array}{l}\text { Odds } \\
\text { Ratio }\end{array}$ & \multicolumn{2}{|c|}{$\begin{array}{l}\text { 95\% Confidence } \\
\text { Limits }\end{array}$} & p-value \\
\hline \multicolumn{9}{|l|}{ Age Category } & \\
\hline$<65$ years & $\mathrm{I}, \mathrm{I} 66,440$ & $52 \%$ & 347 & $57 \%$ & 0.019 & $N / A$ & & & $\mathrm{~N} / \mathrm{A}$ \\
\hline$\geq 65$ years & $\mathrm{I}, 066,702$ & $48 \%$ & 262 & $43 \%$ & & $0.84 I$ & 0.666 & 1.062 & 0.1454 \\
\hline & & & & & & \multicolumn{4}{|c|}{ Reference group $=\mathrm{Age}<65$} \\
\hline \multicolumn{10}{|l|}{ Gender } \\
\hline Male & $1,009,182$ & $45 \%$ & 358 & $59 \%$ & $<0.0001$ & 1.652 & 1.396 & 1.953 & $<0.0001$ \\
\hline Female & $1,223,960$ & $55 \%$ & 251 & $41 \%$ & & $\mathrm{~N} / \mathrm{A}$ & N/A & N/A & N/A \\
\hline & & & & & & \multicolumn{4}{|c|}{ Reference group $=$ Female } \\
\hline \multicolumn{10}{|l|}{ Race } \\
\hline Black & 226,988 & $10 \%$ & 125 & $21 \%$ & $<0.0001$ & 2.069 & 1.679 & 2.551 & $<0.0001$ \\
\hline White & $\mathrm{I}, 775,839$ & $80 \%$ & 422 & $69 \%$ & & N/A & N/A & N/A & N/A \\
\hline Other & 192,258 & $9 \%$ & 52 & $9 \%$ & & 1.062 & 0.792 & 1.424 & 0.6894 \\
\hline \multirow[t]{2}{*}{ Unspecified } & 38,057 & $2 \%$ & 10 & $2 \%$ & & 1.01 & 0.538 & 1.895 & 0.9748 \\
\hline & & & & & & \multicolumn{4}{|c|}{ Reference group $=$ White } \\
\hline \multicolumn{10}{|l|}{ Insurance Type } \\
\hline Medicaid & 213,795 & $10 \%$ & 74 & $12 \%$ & 0.004 & 1.103 & 0.808 & 1.505 & 0.538 \\
\hline Managed Care & 746,579 & $33 \%$ & 183 & $30 \%$ & & 1.045 & 0.815 & 1.339 & 0.7294 \\
\hline Uninsured & 71,561 & $3 \%$ & 32 & $5 \%$ & & 1.562 & 1.032 & 2.363 & 0.0349 \\
\hline Other ins & 90,998 & $4 \%$ & 29 & $5 \%$ & & 1.162 & 0.775 & 1.742 & 0.4684 \\
\hline \multirow[t]{2}{*}{ Medicare } & $1,110,209$ & $50 \%$ & 291 & $48 \%$ & & N/A & N/A & N/A & N/A \\
\hline & & & & & & \multicolumn{4}{|c|}{ Reference group $=$ Managed care } \\
\hline \multicolumn{10}{|l|}{ Admission Type } \\
\hline Elective & $\mathrm{I}, 36 \mathrm{I}, 309$ & $61 \%$ & 279 & $46 \%$ & $<0.0001$ & $N / A$ & $N / A$ & $N / A$ & $N / A$ \\
\hline \multirow[t]{2}{*}{ Urgent/Emergent } & 871,833 & $39 \%$ & 330 & $54 \%$ & & 1.587 & 1.335 & 1.886 & $<0.0001$ \\
\hline & & & & & & \multicolumn{4}{|c|}{ Reference group $=$ Elective } \\
\hline $\begin{array}{l}\text { Hospital } \\
\text { Characteristics }\end{array}$ & & & & & & \multicolumn{4}{|c|}{ Hospital Characteristics } \\
\hline Hospital Region & & & & & & & & & \\
\hline South & $1,060,938$ & $48 \%$ & 303 & $50 \%$ & 0.006 & $N / A$ & $N / A$ & $\mathrm{~N} / \mathrm{A}$ & N/A \\
\hline Northeast & 280,004 & $13 \%$ & 63 & $10 \%$ & & 0.862 & 0.647 & 1.147 & 0.3072 \\
\hline Midwest & 516,277 & $23 \%$ & 117 & $19 \%$ & & 0.868 & 0.696 & 1.083 & 0.2089 \\
\hline West & 375,923 & $17 \%$ & 126 & $21 \%$ & & 1.52 & 1.221 & 1.893 & 0.0002 \\
\hline & & & & & & & eferenc & roup $=$ Sol & \\
\hline Teaching Hospital & & & & & & & & & \\
\hline Teaching Hospital & $1,039,632$ & $47 \%$ & 331 & $54 \%$ & 0.0001 & $\mathrm{I} .107$ & 0.914 & 1.34 & $0.298 I$ \\
\hline Non-Teaching Hospital & $1,193,510$ & $53 \%$ & 278 & $46 \%$ & & $\mathrm{~N} / \mathrm{A}$ & $\mathrm{N} / \mathrm{A}$ & $N / A$ & N/A \\
\hline & & & & & & Refe & nce grou & Teaching & lospital \\
\hline
\end{tabular}


Table I (Continued).

\begin{tabular}{|c|c|c|c|c|c|c|c|c|c|}
\hline \multirow[t]{3}{*}{$\begin{array}{l}\text { Patient } \\
\text { Characteristics }\end{array}$} & \multirow{2}{*}{\multicolumn{2}{|c|}{$\begin{array}{c}\begin{array}{c}\text { Without Difficult } \\
\text { Intubation }\end{array} \\
2,233,142\end{array}$}} & \multirow{2}{*}{\multicolumn{2}{|c|}{$\begin{array}{c}\begin{array}{c}\text { With Difficult } \\
\text { Intubation }\end{array} \\
609\end{array}$}} & \multirow[t]{3}{*}{ p-value ${ }^{a}$} & \multirow{2}{*}{\multicolumn{4}{|c|}{$\begin{array}{c}\text { Comparing Difficult Intubation to No } \\
\text { Difficult Intubation }\end{array}$}} \\
\hline & & & & & & & & & \\
\hline & \multirow[t]{2}{*}{$\begin{array}{l}\text { Number of } \\
\text { Patients }\end{array}$} & $\begin{array}{l}\text { Percent of } \\
\text { Patients }\end{array}$ & $\begin{array}{l}\text { Number of } \\
\text { Patients }\end{array}$ & $\begin{array}{l}\text { Percent of } \\
\text { Patients }\end{array}$ & & $\begin{array}{l}\text { Odds } \\
\text { Ratio }\end{array}$ & \multicolumn{2}{|c|}{$\begin{array}{l}\text { 95\% Confidence } \\
\text { Limits }\end{array}$} & p-value ${ }^{a}$ \\
\hline \multicolumn{9}{|l|}{ Bed Size } & \\
\hline $500+$ beds & 777,012 & $35 \%$ & 305 & $50 \%$ & $<0.0001$ & 1.567 & 1.25 & 1.965 & $<0.0001$ \\
\hline $300-500$ & 671,676 & $30 \%$ & 147 & $24 \%$ & & 0.94 & 0.746 & 1.185 & 0.6014 \\
\hline \multirow[t]{2}{*}{$<300$} & 784,454 & $35 \%$ & 157 & $26 \%$ & & N/A & N/A & N/A & N/A \\
\hline & & & & & & \multicolumn{4}{|c|}{ Reference group $=<300$} \\
\hline \multicolumn{10}{|l|}{ Urban vs Rural } \\
\hline Urban & $2,004,270$ & $90 \%$ & 558 & $92 \%$ & 0.127 & 1.038 & 0.774 & 1.393 & 0.8038 \\
\hline \multirow[t]{2}{*}{ Rural } & 228,872 & $10 \%$ & 51 & $8 \%$ & & N/A & $N / A$ & $\mathrm{~N} / \mathrm{A}$ & N/A \\
\hline & & & & & & \multicolumn{4}{|c|}{ Reference group=Urban } \\
\hline
\end{tabular}

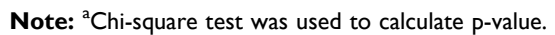

Abbreviation: N/A, not applicable.

Table 2 Distribution of Comorbidities for Patients with and without Difficult Intubation Prior to Risk Adjustment Matching

\begin{tabular}{|c|c|c|c|c|c|c|c|c|c|}
\hline $\begin{array}{l}\text { Patient } \\
\text { Comorbidities }\end{array}$ & $\begin{array}{c}\text { Number } \\
\text { of Patients } \\
\text { without } \\
\text { Difficult } \\
\text { Intubation }\end{array}$ & $\begin{array}{l}\text { Percent of } \\
\text { Patients } \\
\text { without } \\
\text { Difficult } \\
\text { Intubation }\end{array}$ & $\begin{array}{c}\text { Number } \\
\text { of Patients } \\
\text { with } \\
\text { Difficult } \\
\text { Intubation }\end{array}$ & $\begin{array}{c}\text { Percent of } \\
\text { Patients } \\
\text { with } \\
\text { Difficult } \\
\text { Intubation }\end{array}$ & p-value ${ }^{a}$ & $\begin{array}{c}\text { Odds } \\
\text { Ratio }^{b}\end{array}$ & $\begin{array}{c}\text { Lower } 95 \% \\
\text { Confidence } \\
\text { Limits }\end{array}$ & $\begin{array}{c}\text { Upper } 95 \% \\
\text { Confidence } \\
\text { Limits }\end{array}$ & p-value ${ }^{a}$ \\
\hline Obesity & 582,344 & $26 \%$ & 265 & $44 \%$ & $<0.0001$ & 2.202 & 1.857 & 2.612 & $<0.0001$ \\
\hline Cancer & 190,379 & $9 \%$ & 86 & $14 \%$ & $<0.000$ I & 1.886 & 1.458 & 2.44 & $<0.0001$ \\
\hline $\begin{array}{l}\text { Acquired Immune } \\
\text { Deficiency Disease }\end{array}$ & 2,484 & $0 \%$ & 2 & $0 \%$ & 0.108 & 1.654 & 0.41 & 6.67 & 0.4796 \\
\hline Congestive Heart Failure & 265,728 & $12 \%$ & 135 & $22 \%$ & $<0.0001$ & 1.236 & 0.989 & $\mathrm{I} .545$ & 0.0621 \\
\hline $\begin{array}{l}\text { Chronic Obstructive } \\
\text { Pulmonary Disease }\end{array}$ & 408,685 & $18 \%$ & 176 & $29 \%$ & $<0.0001$ & 1.553 & 1.295 & $1.86 \mid$ & $<0.0001$ \\
\hline Cardiovascular Disease & 107,398 & $5 \%$ & 55 & $9 \%$ & $<0.0001$ & 1.419 & 1.054 & 1.911 & 0.0211 \\
\hline Diabetes & 447,307 & $20 \%$ & 177 & $29 \%$ & $<0.000$ I & 1.146 & 0.954 & 1.378 & 0.145 \\
\hline Diabetes with Sequela & 178,372 & $8 \%$ & 107 & $18 \%$ & $<0.0001$ & 1.432 & 1.109 & 1.849 & 0.006 \\
\hline Hypertension & $\mathrm{I}, 389,328$ & $62 \%$ & 452 & $74 \%$ & $<0.0001$ & 1.3 & 1.061 & 1.592 & 0.0114 \\
\hline Metastatic Solid Tumor & 49,571 & $2 \%$ & 24 & $4 \%$ & 0.0039 & 1.123 & 0.712 & 1.772 & 0.6183 \\
\hline Myocardial Infarction & 223,036 & $10 \%$ & 94 & $15 \%$ & $<0.000$ I & 0.988 & 0.777 & 1.255 & 0.9201 \\
\hline $\begin{array}{l}\text { Peripheral Vascular } \\
\text { Disease }\end{array}$ & 173,699 & $8 \%$ & 83 & $14 \%$ & $<0.0001$ & 1.289 & 1.007 & 1.649 & 0.0439 \\
\hline Renal Disease & 250,842 & $11 \%$ & 120 & $20 \%$ & $<0.0001$ & 1.048 & 0.815 & $\mathrm{I} .348$ & 0.7135 \\
\hline Mild Liver Disease & 79,086 & $4 \%$ & 38 & $6 \%$ & 0.0003 & I.I57 & 0.811 & 1.651 & 0.422 \\
\hline $\begin{array}{l}\text { Moderate/Severe Liver } \\
\text { Disease }\end{array}$ & 9,014 & $0 \%$ & 10 & $2 \%$ & $<0.0001$ & 2.344 & 1.193 & 4.606 & 0.0134 \\
\hline Paralysis & 30,879 & $1 \%$ & 20 & $3 \%$ & $<0.0001$ & 1.435 & 0.896 & 2.298 & 0.1332 \\
\hline Dementia & 61,454 & $3 \%$ & 16 & $3 \%$ & 0.8509 & 0.919 & 0.553 & 1.527 & 0.7431 \\
\hline
\end{tabular}

Notes: ${ }^{a}$ Chi-square test was used to calculate p-value. ${ }^{b}$ Reference group for comorbidity odds ratio is patients without the comorbidity for each comorbid condition.

accounted for most differences in the distribution of demographic and hospital characteristics (Table 4) and comorbid conditions (Table 5). The difference between DI and
non-DI matched cohorts for all outcome measures remained significant. Compared to the non-DI patient group, patients in the DI group had $\$ 14,468$ higher mean 


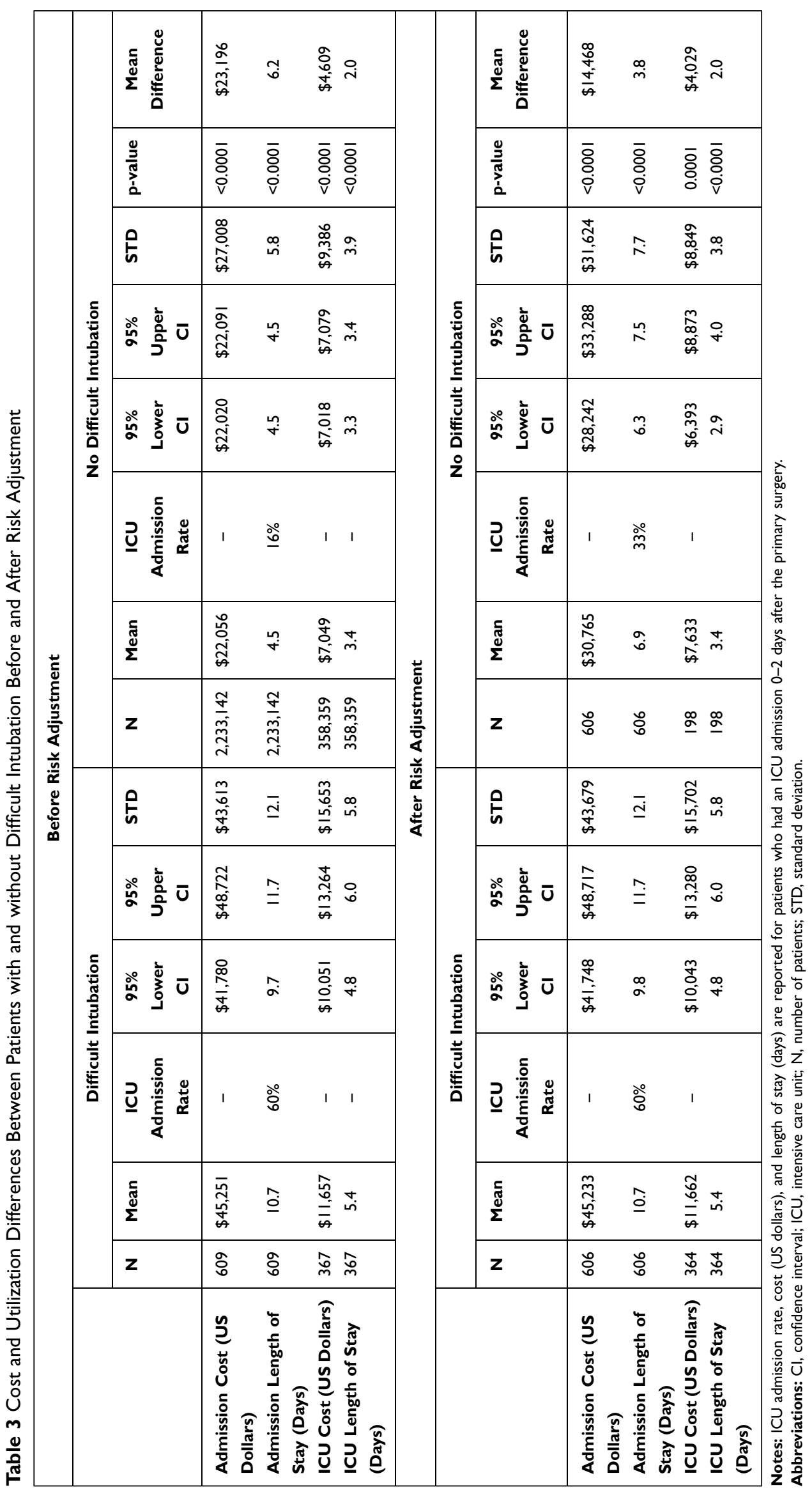


Table 4 Demographic Characteristics of Patients with Difficult Intubation Hospital Inpatient Admissions for Patients After Risk Adjustment Matching

\begin{tabular}{|c|c|c|c|c|c|c|c|c|c|}
\hline \multirow[t]{3}{*}{$\begin{array}{l}\text { Patient } \\
\text { Characteristics }\end{array}$} & \multirow{2}{*}{\multicolumn{2}{|c|}{$\begin{array}{c}\text { Without Difficult } \\
\text { Intubation }\end{array}$}} & \multirow{2}{*}{\multicolumn{2}{|c|}{$\begin{array}{c}\text { With Difficult } \\
\text { Intubation }\end{array}$}} & \multirow[t]{3}{*}{ p-value ${ }^{a}$} & \multicolumn{4}{|c|}{$\begin{array}{c}\text { Comparing Difficult Intubation to } \\
\text { No Difficult Intubation }\end{array}$} \\
\hline & & & & & & \multirow[b]{2}{*}{$\begin{array}{l}\text { Odds } \\
\text { Ratio }\end{array}$} & \multicolumn{2}{|c|}{$\begin{array}{l}\text { 95\% Confidence } \\
\text { Limits }\end{array}$} & \multirow[b]{2}{*}{ p-value ${ }^{a}$} \\
\hline & $\begin{array}{c}\text { Number of } \\
\text { Patients }\end{array}$ & $\begin{array}{l}\text { Percent of } \\
\text { Patients }\end{array}$ & $\begin{array}{l}\text { Number of } \\
\text { Patients }\end{array}$ & $\begin{array}{l}\text { Percent of } \\
\text { Patients }\end{array}$ & & & $\begin{array}{l}\text { Lower } \\
\text { Limit }\end{array}$ & $\begin{array}{l}\text { Upper } \\
\text { Limit }\end{array}$ & \\
\hline \multicolumn{10}{|l|}{ Age Category } \\
\hline$<65$ years & 324 & $53 \%$ & 346 & $57 \%$ & 0.204 & N/A & & & $\mathrm{N} / \mathrm{A}$ \\
\hline \multirow[t]{2}{*}{$\geq 65$ years } & 282 & $47 \%$ & 260 & $43 \%$ & & 0.948 & 0.676 & 1.331 & 0.7591 \\
\hline & & & & & & \multicolumn{4}{|c|}{ Reference group $=\mathrm{Age}<65$} \\
\hline \multicolumn{10}{|l|}{ Gender } \\
\hline Male & 359 & $59 \%$ & 356 & $59 \%$ & 0.861 & 0.994 & 0.783 & 1.262 & 0.9595 \\
\hline \multirow[t]{2}{*}{ Female } & 247 & $41 \%$ & 250 & $41 \%$ & & N/A & $\mathrm{N} / \mathrm{A}$ & N/A & N/A \\
\hline & & & & & & \multicolumn{4}{|c|}{ Reference group $=$ Female } \\
\hline \multicolumn{10}{|l|}{ Race } \\
\hline Black & 114 & $19 \%$ & 125 & $21 \%$ & 0.691 & 1.162 & 0.854 & $\mathrm{I} .582$ & 0.3384 \\
\hline White & 437 & $72 \%$ & 420 & $69 \%$ & & $\mathrm{~N} / \mathrm{A}$ & $\mathrm{N} / \mathrm{A}$ & $\mathrm{N} / \mathrm{A}$ & N/A \\
\hline Other & 48 & $8 \%$ & 51 & $8 \%$ & & 1.145 & 0.738 & 1.775 & 0.5456 \\
\hline \multirow[t]{2}{*}{ Unspecified } & 7 & $1 \%$ & 10 & $2 \%$ & & 1.453 & 0.532 & 3.971 & 0.4661 \\
\hline & & & & & & \multicolumn{4}{|c|}{ Reference group $=$ White } \\
\hline \multicolumn{10}{|l|}{ Insurance Type } \\
\hline Medicaid & 87 & $14 \%$ & 74 & $12 \%$ & 0.293 & 0.822 & 0.528 & 1.279 & 0.3856 \\
\hline Managed Care & 149 & $25 \%$ & 182 & $30 \%$ & & 1.192 & 0.832 & $\mathrm{I} .709$ & 0.3392 \\
\hline Uninsured & 34 & $6 \%$ & 32 & $5 \%$ & & 0.958 & 0.534 & 1.72 & 0.8863 \\
\hline Other ins & 29 & $5 \%$ & 29 & $5 \%$ & & 1.036 & 0.578 & 1.855 & 0.9065 \\
\hline \multirow[t]{2}{*}{ Medicare } & 307 & $51 \%$ & 289 & $48 \%$ & & N/A & $\mathrm{N} / \mathrm{A}$ & $N / A$ & $\mathrm{~N} / \mathrm{A}$ \\
\hline & & & & & & \multicolumn{4}{|c|}{ Reference group $=$ Managed care } \\
\hline \multicolumn{10}{|l|}{ Admission Type } \\
\hline Elective & 272 & $45 \%$ & 277 & $46 \%$ & 0.773 & $\mathrm{~N} / \mathrm{A}$ & $\mathrm{N} / \mathrm{A}$ & $\mathrm{N} / \mathrm{A}$ & $\mathrm{N} / \mathrm{A}$ \\
\hline \multirow[t]{2}{*}{ Urgent/Emergent } & 334 & $55 \%$ & 329 & $54 \%$ & & 0.951 & 0.743 & 1.217 & 0.688 \\
\hline & & & & & & \multicolumn{4}{|c|}{ Reference group $=$ Elective } \\
\hline \multicolumn{10}{|l|}{ Hospital Characteristics } \\
\hline \multicolumn{10}{|l|}{ Hospital Region } \\
\hline South & 325 & $54 \%$ & 301 & $50 \%$ & 0.495 & $\mathrm{~N} / \mathrm{A}$ & $\mathrm{N} / \mathrm{A}$ & N/A & $\mathrm{N} / \mathrm{A}$ \\
\hline Northeast & 59 & $10 \%$ & 62 & $10 \%$ & & 1.178 & 0.771 & 1.802 & 0.4486 \\
\hline Midwest & 100 & $17 \%$ & 117 & $19 \%$ & & 1.287 & 0.926 & $\mathrm{I} .788$ & 0.1338 \\
\hline West & 122 & $20 \%$ & 126 & $21 \%$ & & 1.126 & 0.821 & 1.545 & 0.462 \\
\hline & & & & & & & Reference & roup $=$ So & \\
\hline Teaching Hospital & & & & & & & & & \\
\hline Teaching Hospital & 347 & $57 \%$ & 330 & $54 \%$ & 0.325 & 0.884 & 0.667 & 1.17 & 0.3869 \\
\hline Non-Teaching Hospital & 259 & $43 \%$ & 276 & $46 \%$ & & $\mathrm{~N} / \mathrm{A}$ & $\mathrm{N} / \mathrm{A}$ & $\mathrm{N} / \mathrm{A}$ & N/A \\
\hline & & & & & & & eference & oup $=$ Teac & \\
\hline
\end{tabular}

(Continued) 
Table 4 (Continued).

\begin{tabular}{|c|c|c|c|c|c|c|c|c|c|}
\hline \multirow[t]{3}{*}{$\begin{array}{l}\text { Patient } \\
\text { Characteristics }\end{array}$} & \multirow{2}{*}{\multicolumn{2}{|c|}{$\begin{array}{c}\begin{array}{c}\text { Without Difficult } \\
\text { Intubation }\end{array} \\
606\end{array}$}} & \multirow{2}{*}{\multicolumn{2}{|c|}{$\begin{array}{c}\text { With Difficult } \\
\text { Intubation }\end{array}$}} & \multirow[t]{3}{*}{ p-value ${ }^{a}$} & \multicolumn{4}{|c|}{$\begin{array}{c}\text { Comparing Difficult Intubation to } \\
\text { No Difficult Intubation }\end{array}$} \\
\hline & & & & & & \multirow[b]{2}{*}{$\begin{array}{l}\text { Odds } \\
\text { Ratio }\end{array}$} & \multicolumn{2}{|c|}{$\begin{array}{c}\text { 95\% Confidence } \\
\text { Limits }\end{array}$} & \multirow[b]{2}{*}{ p-value } \\
\hline & $\begin{array}{l}\text { Number of } \\
\text { Patients }\end{array}$ & $\begin{array}{l}\text { Percent of } \\
\text { Patients }\end{array}$ & $\begin{array}{l}\text { Number of } \\
\text { Patients }\end{array}$ & $\begin{array}{l}\text { Percent of } \\
\text { Patients }\end{array}$ & & & $\begin{array}{l}\text { Lower } \\
\text { Limit }\end{array}$ & $\begin{array}{l}\text { Upper } \\
\text { Limit }\end{array}$ & \\
\hline \multicolumn{10}{|l|}{ Bed Size } \\
\hline $500+$ beds & 319 & $53 \%$ & 303 & $50 \%$ & 0.638 & 1.027 & 0.735 & 1.435 & 0.8758 \\
\hline $300-500$ & $|4|$ & $23 \%$ & 146 & $24 \%$ & & 0.99 & 0.705 & 1.39 & 0.9526 \\
\hline \multirow[t]{2}{*}{$<300$} & 146 & $24 \%$ & 157 & $26 \%$ & & $\mathrm{~N} / \mathrm{A}$ & $\mathrm{N} / \mathrm{A}$ & $\mathrm{N} / \mathrm{A}$ & N/A \\
\hline & & & & & & \multicolumn{4}{|c|}{ Reference group $=<300$} \\
\hline \multicolumn{10}{|l|}{ Urban vs Rural } \\
\hline Urban & 558 & $92 \%$ & 555 & $92 \%$ & 0.753 & 0.939 & 0.609 & $\mathrm{I} .447$ & 0.7753 \\
\hline Rural & 48 & $8 \%$ & 51 & $8 \%$ & & $N / A$ & $\mathrm{~N} / \mathrm{A}$ & $\mathrm{N} / \mathrm{A}$ & N/A \\
\hline & & & & & & \multicolumn{4}{|c|}{ Reference group $=$ Urban } \\
\hline
\end{tabular}

Note: ${ }^{a}$ Chi-square test was used to calculate $p$-value.

Abbreviation: N/A, not applicable.

cost of hospital inpatient stay $(\$ 45,233 \pm \$ 43,679$ vs. $\$ 30,765 \pm \$ 31,624) ; 3.8$ days longer $\operatorname{LOS}(10.7 \pm 12.1$ days vs. $6.9 \pm 7.7$ days); higher post-operative ICU admission rate $(60 \%$ vs. $33 \%)$; $\$ 4029$ higher ICU costs $(\$ 11,662$ $\pm \$ 15,702$ vs. $\$ 7,633 \pm \$ 8,849)$; and 2.0 days longer length of stay in the ICU $(5.4 \pm 5.8$ days vs. $3.4 \pm 3.8$ days $)$. All p-values were $<0.0001$ (Table 3 ).

\section{Factors Associated with Documented DI}

Both patient characteristics and hospital characteristics were independently associated with the likelihood of documented DI. Compared to patients who were white, female, or having elective surgical procedure, the odds of being in the DI group were 2.1 times higher for black patients (OR: 2.1, 95\% CI: 1.7-2.6; $\mathrm{p}<0.0001)$; 1.7 times higher for male patients (OR: 1.7, 95\% CI: 1.4-2.0, p<0.0001); and 1.6 times higher for patients with urgent or emergent admission (OR: 1.6; 95\% CI: 1.3-1.9, p<0.0001) (Table 1). Additionally, when evaluating the risk associated with specific chronic conditions, the risk was higher for patients with the chronic condition than for patients without the specific condition. The risk was 2.2 times higher for patients with obesity (OR: 2.2; 95\% CI: 1.9-2.6; $\mathrm{p}<0.0001) ; 1.9$ times higher for patients with cancer (OR: 1.9; 95\% CI: 1.5-2.4; $\mathrm{p}<0.0001$ ); 1.6 times higher for patients with COPD (OR: 1.6; 95\% CI: 1.3-1.9; $\mathrm{p}<0.0001)$; and 1.4 times higher for patients with cardiovascular disease (OR: 1.4; 95\% CI: 1.1-1.9; $\mathrm{p}=0.02$ ) (Table 2). Hospital factors included hospital size and US Census Region. Compared to patients in hospitals with fewer than 300 beds, patients at hospitals with more than 500 beds were 1.6 times more likely to have documented DI cases (OR: 1.6, 95\% CI: 1.3-2.0; p<0.0001) and patients at hospitals in the West region were 1.5 times more likely to have documented DI cases compared to hospitals in the South region (OR: 1.5, 95\% CI: 1.2-1.9, $\mathrm{p}<0.0001$ ) (Table 1).

\section{Discussion}

Airway management is fundamental to safe anesthetic practice. Difficult intubation, failed intubation, and wrongly placed tracheal tubes account for $2.3 \%$ of all anesthesia-related deaths. ${ }^{13}$ Problems with tracheal intubation were the most frequently recorded primary airway problem, which accounted for $39 \%$ of all events during anesthesia. ${ }^{9}$ To the best of our knowledge, this is the first study to use ICD-10-CM diagnosis codes to identify documented DI and explore factors associated with DI using Real World Data (RWD), and to examine the difference in healthcare utilization between DI and non-DI patient groups. This new information highlights the significant cost burden associated with DI. The variance in both cost and length of stay measures was high in the DI and non-DI groups, suggesting that while the average patient in the DI 
Table 5 Patient and Hospital Demographic Characteristics and Logistic Regression Outputs After Risk Adjustment Matching

\begin{tabular}{|c|c|c|c|c|c|c|c|c|c|}
\hline $\begin{array}{l}\text { Patient } \\
\text { Comorbidities }\end{array}$ & $\begin{array}{c}\text { Number of } \\
\text { Patients } \\
\text { without } \\
\text { Difficult } \\
\text { Intubation }\end{array}$ & $\begin{array}{l}\text { Percent of } \\
\text { Patients } \\
\text { without } \\
\text { Difficult } \\
\text { Intubation }\end{array}$ & $\begin{array}{c}\text { Number } \\
\text { of Patients } \\
\text { with } \\
\text { Difficult } \\
\text { Intubation }\end{array}$ & $\begin{array}{l}\text { Percent of } \\
\text { Patients } \\
\text { with } \\
\text { Difficult } \\
\text { Intubation }\end{array}$ & p-value ${ }^{a}$ & $\begin{array}{l}\text { Odds } \\
\text { Ratio }^{\text {b }}\end{array}$ & $\begin{array}{c}\text { Lower } 95 \% \\
\text { Confidence } \\
\text { Limits }\end{array}$ & $\begin{array}{c}\text { Upper 95\% } \\
\text { Confidence } \\
\text { Limits }\end{array}$ & p-value \\
\hline Obesity & 217 & $36 \%$ & 264 & $44 \%$ & 0.0058 & 1.251 & 0.975 & 1.606 & 0.0781 \\
\hline Cancer & 97 & $16 \%$ & 86 & $14 \%$ & 0.3775 & 0.96 & 0.665 & 1.386 & 0.8268 \\
\hline $\begin{array}{l}\text { Acquired Immune } \\
\text { Deficiency Disease }\end{array}$ & - & $0 \%$ & 2 & $0 \%$ & 0.157 & $N / A$ & N/A & N/A & 0.9794 \\
\hline Congestive Heart Failure & 119 & $20 \%$ & 133 & $22 \%$ & 0.3217 & 1.16 & 0.845 & 1.594 & 0.3579 \\
\hline $\begin{array}{l}\text { Chronic Obstructive } \\
\text { Pulmonary Disease }\end{array}$ & 180 & $30 \%$ & 175 & $29 \%$ & 0.7523 & 0.948 & 0.73 & 1.232 & 0.6907 \\
\hline Cardiovascular Disease & 59 & $10 \%$ & 54 & $9 \%$ & 0.6213 & 0.877 & 0.571 & 1.35 & 0.5518 \\
\hline Diabetes & 147 & $24 \%$ & 176 & $29 \%$ & 0.0596 & 1.201 & 0.909 & 1.587 & 0.1979 \\
\hline Diabetes with Sequela & 85 & $14 \%$ & 106 & $17 \%$ & 0.0978 & 1.242 & 0.843 & 1.828 & 0.2727 \\
\hline Hypertension & 447 & $74 \%$ & 450 & $74 \%$ & 0.8442 & 0.947 & 0.707 & 1.268 & $0.7|4|$ \\
\hline Metastatic Solid Tumor & 32 & $5 \%$ & 24 & $4 \%$ & 0.2737 & 0.788 & 0.424 & 1.465 & 0.4517 \\
\hline Myocardial Infarction & 85 & $14 \%$ & 94 & $16 \%$ & 0.4662 & 1.109 & 0.788 & 1.562 & 0.553 \\
\hline $\begin{array}{l}\text { Peripheral Vascular } \\
\text { Disease }\end{array}$ & 92 & $15 \%$ & 83 & $14 \%$ & 0.462 & 0.87 & 0.614 & 1.232 & 0.4315 \\
\hline Renal Disease & 116 & $19 \%$ & 120 & $20 \%$ & 0.7717 & 0.915 & 0.642 & 1.304 & 0.6234 \\
\hline Mild Liver Disease & 35 & $6 \%$ & 38 & $6 \%$ & 0.7172 & 1.244 & 0.724 & 2.14 & 0.429 \\
\hline $\begin{array}{l}\text { Moderate/Severe Liver } \\
\text { Disease }\end{array}$ & 16 & $3 \%$ & 10 & $2 \%$ & 0.2342 & 0.556 & 0.226 & 1.365 & 0.2003 \\
\hline Paralysis & 19 & $3 \%$ & 20 & $3 \%$ & 0.8707 & 1.188 & 0.583 & 2.418 & 0.6358 \\
\hline Dementia & 19 & $3 \%$ & 16 & $3 \%$ & 0.6068 & 0.918 & 0.449 & 1.878 & 0.8145 \\
\hline
\end{tabular}

Notes: ${ }^{a}$ Chi-square test was used to calculate $\mathrm{p}$-value. ${ }^{\mathrm{b}}$ Reference group for comorbidity odds ratio is patients without the comorbidity for each comorbid condition. Abbreviation: N/A, not applicable.

group experiences higher costs and length of stay compared to the average patient in the non-DI group, at the individual patient level, these differences may be more difficult to detect.

Our finding of obesity as a risk factor for DI is consistent with other studies which have indicated that patients with obesity were at increased risk of an adverse airway event. ${ }^{9,14-16}$ Reasons for this include mechanical difficulty in securing the airway, increased risk of aspiration, increased risk of airway obstruction during intubation, and accelerated speed and extent of oxygen desaturation during airway obstruction. ${ }^{17-19}$ The current study also suggests that patient gender, race, and other chronic conditions are associated with risk of DI. This is consistent with previous reports that increasing age, American Society of Anesthesiologists physical status, and CCI are associated with failed and difficult intubation. ${ }^{20,21}$ Importantly, many of the factors associated with DI in our study are linked to obesity, including diabetes, cardiovascular disease, cancer, and liver disease. ${ }^{22-25}$ Further prospective research is required to determine whether some of the chronic conditions are directly related to DI, or if they are confounding factors associated with a major predictor of DI, such as obesity. Other conditions, including the presence of head and neck cancer, arthritis, ankylosing spondylitis, and diabetes with limited joint mobility, which can alter the patient's ability to lie flat are also associated with failed and difficult intubation, due to narrowing of the airway and challenges in positioning during intubation. ${ }^{26-28}$ In addition, large hospitals with $>500$ beds were more likely to have documented DI cases, which may reflect more consistent reporting standards often instituted at large hospitals. Although risk factors associated with DI have been identified, prediction of DI is a challenging task, and additional research to understand the predictors for DI would be beneficial, allowing clinicians to choose the appropriate method and device for patient airway management in challenging cases. ${ }^{16,29-31}$

\section{Implications and Future Actions}

DI may occur in any type of surgical procedure and it can be costly when it happens. Our research highlights a need for clinicians who perform tracheal intubations to carefully consider options that could improve the chances of 
successful intubation on the first attempt. The advances in new technology in tracheal intubation in a hospital setting may provide alternative options. For example, several studies demonstrated the benefits of video laryngoscopy, including a shorter time required for tracheal intubation, a higher rate of successful intubations, and a reduced need for external manipulation of the larynx or other maneuvers in the case of an expected DI. ${ }^{32-35}$ The benefits and costs associated with alternative technologies should be considered through future research.

The use of administrative data for retrospective analysis has both strengths and weaknesses. Due to the large number of hospitals, we have a good representation of hospitals in all regions of the United States, as well as good representation of patients with different types of insurance coverage. However, we expect the administrative data to suffer significant under-documentation of DI in the medical claims data. Only $0.03 \%$ of admissions in this study were found to have documented DI. Elsewhere, the reported incidence of DI has been estimated to be $1.5-8.5 \%$, while failed intubation occurred in $0.13-0.3 \%$ of the general population. ${ }^{36}$ This discrepancy between expected and documented DI suggests that in our analysis, some admissions with DI may be assigned to the cohort assumed to have no DI. It is also possible that admissions that have documented DI have more serious complications resulting from DI than admissions that may have experienced undocumented DI. Therefore, the cost differential identified in the current study may be a conservative estimate overall, but not generalizable beyond cases of documented DI. Finally, our analysis did not include access to patient electronic medical records, limiting our ability to evaluate patient clinical characteristics to the data available in the administrative dataset.

\section{Conclusion}

In conclusion, the current study demonstrates the significant cost burden averaging $\$ 14,468$ and increased postoperative LOS averaging 3.8 days for patients with documented difficult tracheal intubation.

\section{Abbreviations}

DI, difficult intubation; CFR, Code Of Federal Regulations; CHF, congestive heart failure; CI, confidence interval; CICV, can't intubate can't ventilate; COPD, chronic obstructive pulmonary disease; HIPAA, Health Insurance Portability and Accountability Act; ICD-10-CM, International Classification Of Diseases, Tenth Edition, Clinical Modification; ICU, intensive care unit; IRB, Institutional Review Board; LOS, length of stay; MDC, Major Diagnostic Category; MS-DRG, Medicare Severity-Diagnosis Related Group; RWD, real world data; US, United States.

\section{Data Sharing Statement}

The data that support the findings of this study are available from Premier Inc. but restrictions apply to the availability of these data, which were used under license for the current study, and so are not publicly available. Data may be available from the authors upon reasonable request and with permission of Premier Inc.

\section{Ethics Approval and Informed Consent}

This is a retrospective cohort study using medical billing/ encounter data. No registry was used in current study. The data we used are de-identified and complied with Health Insurance Portability and Accountability Act (HIPAA) confidentiality requirements. Per US Title 45 CFR (Code of Federal Regulations) Part 46.101, institutional review board (IRB) approval for this study was not required under the exemption that this research involved the study of existing data and that the information was recorded in such a manner that the subjects could not be identified, directly or through identifiers linked to the subjects.

\section{Author Contributions}

All authors made substantial contributions to conception and design, acquisition of data, or analysis and interpretation of data; took part in drafting the article or revising it critically for important intellectual content; agreed to submit to the current journal; gave final approval of the version to be published; and agree to be accountable for all aspects of the work.

\section{Funding}

There is no funding to report.

\section{Disclosure}

All 3 authors are paid employees of Medtronic. The authors report no other conflicts of interest in this work.

\section{References}

1. Cook TM, MacDougall-Davis SR. Complications and failure of airway management. Br J Anaesth. 2012;109(Suppl 1):i68-i85. doi:10.1093/bja/aes393

2. Kriege M, Alflen C, Tzanova I, Schmidtmann I, Piepho T, Noppens RR. Evaluation of the McGrath MAC and macintosh laryngoscope for tracheal intubation in 2000 patients undergoing general anaesthesia: the randomised multicentre EMMA trial study protocol. BMJ Open. 2017;7(8):e016907. doi:10.1136/bmjopen-2017-016907 
3. Norskov AK, Rosenstock CV, Wetterslev J, Astrup G, Afshari A, Lundstrom LH. Diagnostic accuracy of anaesthesiologists' prediction of difficult airway management in daily clinical practice: a cohort study of 188064 patients registered in the Danish anaesthesia database. Anaesthesia. 2015;70(3):272-281.

4. Mallampati SR, Gatt SP, Gugino LD, et al. A clinical sign to predict difficult tracheal intubation; a prospective study. Can Anaesth Soc J. 1985;32(4):429-434. doi:10.1007/BF03011357

5. Wang CMZ, Pang KP, Tan SG, et al. Predictors of difficulty in intubation in patients with obstructive sleep apnoea. Med J Malaysia. 2019;74(2):133-137.

6. Zadrobilek E. The cormack-lehane classification: twenty-fifth anniversary of the first published description. Internet J Airway Manage. 2009;5.

7. Adnet F, Borron SW, Racine SX, et al. The intubation difficulty scale (IDS): proposal and evaluation of a new score characterizing the complexity of endotracheal intubation. Anesthesiology. 1997;87 (6):1290-1297. doi:10.1097/00000542-199712000-00005

8. Krage R, van Rijn C, van Groeningen D, Loer SA, Schwarte LA, Schober P. Cormack-lehane classification revisited. Br J Anaesth. 2010;105(2):220-227. doi:10.1093/bja/aeq136

9. Levitan RM, Everett WW, Ochroch EA. Limitations of difficult airway prediction in patients intubated in the emergency department. Ann Emerg Med. 2004;44(4):307-313. doi:10.1016/j.annemergmed.2004.05.006

10. Premier Applied Sciences ${ }^{\circledR}$. Premier Healthcare Database White Paper: Data That Informs and Performs. Premier Inc.; March 2, 2020.

11. Bursac Z, Gauss CH, Williams DK, Hosmer DW. Purposeful selection of variables in logistic regression. Source Code Biol Med. 2008;3 (1):17. doi:10.1186/1751-0473-3-17

12. Bursac Z, Gauss C, Williams D, Hosmer D. A purposeful selection of variables macro for logistic regression. J Source Code Biol Med. 2007;3:173-2007.

13. Li G, Warner M, Lang BH, Huang L, Sun LS. Epidemiology of anesthesia-related mortality in the United States, 1999-2005. Anesthesiology. 2009;110(4):759-765. doi:10.1097/ALN.0b013e31819b5bdc

14. Juvin P, Lavaut E, Dupont $\mathrm{H}$, et al. Difficult tracheal intubation is more common in obese than in lean patients. Anesth Analg. 2003;97 (2):595-600. doi:10.1213/01.ANE.0000072547.75928.B0

15. De Jong A, Molinari N, Pouzeratte Y, et al. Difficult intubation in obese patients: incidence, risk factors, and complications in the operating theatre and in intensive care units. BJA. 2014;114 (2):297-306. doi:10.1093/bja/aeu373

16. Endlich Y, Lee J, Culwick MD. Difficult and failed intubation in the first 4000 incidents reported on webAIRS. Anaesth Intensive Care. 2020;48(6):477-487. doi:10.1177/0310057X20957657

17. Kheterpal S, Han R, Tremper KK, et al. Incidence and predictors of difficult and impossible mask ventilation. Anesthesiology. 2006;105 (5):885-891. doi:10.1097/00000542-200611000-00007

18. Kristensen MS. Airway management and morbid obesity. Eur J Anaesthesiol. 2010;27(11):923-927. doi:10.1097/EJA.0b013e32833 d91 aa

19. Farmery AD, Roe PG. A model to describe the rate of oxyhaemoglobin desaturation during apnoea. Br J Anaesth. 1996;76(2):284-291. doi:10.1093/bja/76.2.284

20. Joffe AM, Aziz MF, Posner KL, Duggan LV, Mincer SL, Domino KB. Management of difficult tracheal intubation: a closed claims analysis. Anesthesiology. 2019;131(4):818-829. doi:10.1097/ ALN.0000000000002815
21. Schnittker R, Marshall SD, Berecki-Gisolf J. Patient and surgery factors associated with the incidence of failed and difficult intubation. Anaesthesia. 2020;75(6):756-766. doi:10.1111/anae.14 997

22. Khaodhiar L, McCowen KC, Blackburn GL. Obesity and its comorbid conditions. Clin Cornerstone. 1999;2(3):17-31. doi:10.1016/S10 98-3597(99)90002-9

23. Chu D-T, Minh Nguyet NT, Dinh TC, et al. An update on physical health and economic consequences of overweight and obesity. Diabetes Metab Syndr. 2018;12(6):1095-1100. doi:10.1016/j.dsx.20 18.05.004

24. Fabbrini E, Sullivan S, Klein S. Obesity and nonalcoholic fatty liver disease: biochemical, metabolic, and clinical implications. Hepatology. 2010;51(2):679-689. doi:10.1002/hep.23280

25. Polyzos SA, Kountouras J, Mantzoros CS. Obesity and nonalcoholic fatty liver disease: from pathophysiology to therapeutics. Metabolism. 2019;92:82-97. doi:10.1016/j.metabol.2018.11.014

26. Ahmed-Nusrath A. Anaesthesia for head and neck cancer surgery. BJA Educ. 2017;17(12):383-389. doi:10.1093/bjaed/mkx028

27. Crawley S, Dalton A. Predicting the difficult airway. BJA Educ. 2014;15(5):253-257. doi:10.1093/bjaed/mku047

28. Nowcid L, Valentine E. Difficult airway in diabetes. Transl Perioper Pain Med. 2015;2(1):19-21.

29. Vannucci A, Cavallone LF. Bedside predictors of difficult intubation: a systematic review. Minerva Anestesiol. 2016;82(1):69-83.

30. Detsky ME, Jivraj N, Adhikari NK, et al. Will this patient be difficult to intubate?: the rational clinical examination systematic review. JAMA. 2019;321(5):493-503. doi:10.1001/jama.2018.21413

31. Onal O, Gumus I, Ozdemirkan A, et al. A comparative randomized trial of intubation success in difficult intubation cases: the use of a frova intubation catheter versus a bonfils intubation fiberoscope. Wideochir Inne Tech Maloinwazyjne. 2019;14(4):486-494. doi:10. 5114/wiitm.2019.83610

32. Jungbauer A, Schumann M, Brunkhorst V, Borgers A, Groeben H. Expected difficult tracheal intubation: a prospective comparison of direct laryngoscopy and video laryngoscopy in 200 patients. $\mathrm{Br}$ J Anaesth. 2009;102(4):546-550. doi:10.1093/bja/aep013

33. Aziz MF, Dillman D, Fu R, Brambrink AM. Comparative effectiveness of the C-MAC video laryngoscope versus direct laryngoscopy in the setting of the predicted difficult airway. Anesthesiology. 2012;116 (3):629-636. doi:10.1097/ALN.0b013e318246ea34

34. Asai T M.D., Ph.D, Liu EH M.D., F.R.C.A., Matsumoto S M.D., et al. Use of the pentax-AWS ${ }^{\circledR}$ in 293 patients with difficult airways. Anesthesiology. 2009;110(4):898-904. doi:10.1097/ALN.0b013e318 $19 \mathrm{c} 45 \mathrm{e} 5$

35. Pieters BMA, Maas EHA, Knape JTA, van Zundert AAJ. Videolaryngoscopy vs. direct laryngoscopy use by experienced anaesthetists in patients with known difficult airways: a systematic review and meta-analysis. Anaesthesia. 2017;72(12):1532-1541. doi:10.1111/anae.14057

36. Crosby ET, Cooper RM, Douglas MJ, et al. The unanticipated difficult airway with recommendations for management. Can J Anaesth. 1998;45(8):757-776. doi:10.1007/BF03012147 


\section{Publish your work in this journal}

ClinicoEconomics and Outcomes Research is an international, peerreviewed open-access journal focusing on Health Technology Assessment, Pharmacoeconomics and Outcomes Research in the areas of diagnosis, medical devices, and clinical, surgical and pharmacologica intervention. The economic impact of health policy and health systems organization also constitute important areas of coverage. The manuscript management system is completely online and includes a very quick and fair peer-review system, which is all easy to use. Visit http://www.dovepress.com/testimonials.php to read real quotes from published authors.

Submit your manuscript here: https://www.dovepress.com/clinicoeconomics-and-outcomes-research-journal 